\title{
Incorporating GOMS Analysis into the Design of an EEG Data Visual Analysis Tool
}

\author{
Hua Guo* Diem Tran ${ }^{\dagger} \quad$ David H. Laidlaw \\ Department of Computer Science \\ Brown University
}

\begin{abstract}
In this paper, we present a case study where we incorporate GOMS (Goals, Operators, Methods, and Selectors) [2] task analysis into the design process of a visual analysis tool. We performed GOMS analysis on an Electroencephalography (EEG) analyst's current data analysis strategy to identify important user tasks and unnecessary user actions in his current workflow. We then designed an EEG data visual analysis tool based on the GOMS analysis result. Evaluation results show that the tool we have developed, EEGVis, allows the user to analyze EEG data with reduced subjective cognitive load, faster speed and increased confidence in the analysis quality. The positive evaluation results suggest that our design process demonstrates an effective application of GOMS analysis to discover opportunities for designing better tools to support the user's visual analysis process.
\end{abstract}

Keywords: Human factors, user-centered design.

\section{INTRODUCTION}

In this paper, we present a case study where we perform task analysis to inform the design of an EEG data visual analysis tool. The contribution of this work includes 1) demonstration of the value of GOMS analysis for visual analytics by applying it in the design of EEGVis as well as a brief discussion of its limitation, 2) the design, implementation and evaluation of an EEG data visual analysis tool and 3) an account of the integrated EEG data analysis activity.

EEG is the recording of electrical activity along the scalp, and usually an EEG dataset is collected from multiple electrodes over a time period. Visualization of EEG data commonly falls into one of two categories: visualizing the change in electrical signals on one electrode over time, or visualizing the voltage or power of the EEG data over the whole brain as a topographical map. The tool we have developed aims to support integrated analysis of both types of EEG visualization from different electrodes and time periods.

While GOMS is often applied to estimate task completion time given a user interface, we apply the model in the initial design phase. We hypothesized that this would allow us to identify both tasks that are frequently performed and essential to the user's analysis and wasted user actions imposed by the tools used in the user's current approach. We could then design a tool that offers better support for the important tasks and reduces unnecessary user actions.

\section{Related Work}

Existing works on visual analytic activity decomposition often aim to identify common tasks for general analytic activities, while our work aims at decomposing a specific high-level analysis activity based on analyzing how it is performed by users.

\footnotetext{
*e-mail: hua_guo@brown.edu

†e-mail: diem_tran@brown.edu

†e-mail:dhl@cs.brown.edu
}

Various visualization task taxonomies have been proposed by Wehrend and Lewis [5], Shneiderman [4], Amar et.al [1] and Lee et.al [3]. While task taxonomies could serve as guidelines when choosing interaction or visualization features to be included in the design, they may not always help answer more intricate questions such as how to prioritize support for various tasks or how to analyze in detail how a new design will improve upon an existing one.

Our tool builds on top of EEGLab [6], a toolbox for Matlab that provides EEG data processing and visualization capabilities. While toolboxes like EEGLab allow a user to easily create either a temporal signal visualization or a topographic map from an EEG dataset, our tool might be one of the first tools designed to facilitate integrated analysis of both types of EEG data visualization.

\section{Methods}

We first performed a Card, Moran and Newell GOMS (CMNGOMS) [2] analysis, the original version of GOMS analysis, on the user's workflow based on user interviews and observation. We then designed a visual analysis tool, EEGVis, to support core user tasks and reduce wasted user actions identified through the GOMS analysis. Evaluation of the tool is based on a user study with two expert users, where we used both a subjective cognitive load questionnaire and subjective feedback.

\subsection{GOMS Task Analysis for EEG Data Analysis}

To perform the GOMS analysis, we interviewed a brain scientist and observed him analyzing EEG data. The high-level goal of the brain scientist is to look for interesting brain activation patterns that may lead to novel hypotheses about functionalities of various brain regions. To achieve this goal, the brain scientist works with both temporal signal visualizations and topographical maps from 64 electrodes, over 1000 time points and multiple experiment conditions. In the brain scientist's current workflow, he writes scripts using Matlab and EEGLab to render separate visualizations and adjust the parameters by modifying the scripts. We created a GOMS model to capture the goals and "operators", i.e. actions performed to achieve the goals, involved in this activity, as shown in figure 1.

\subsection{Design of EEGVis}

Based on the GOMS model, we designed the user interface of EEGVis to support important user tasks and reduce unnecessary actions identified in the user's standard workflow. To make it less mentally demanding to perform COMPARE-TIME-SIGNALS and COMPARE-SPATIAL-ACTIVATION-PATTERNS, the distance between two time signals and between two topographical maps are minimized. We added a history list to facilitate the task MARK-INTERESTINGPATTERN and an electrode number-name mapping to remove LOOKUP-ELECTRODE-NUMBER. The input fields are placed close to the visualization to reduce the amount of eye movement actions needed between the inputs and the visualizations. Figure 1 compares the task model for our user's standard workflow and that with EEGVis.

\subsection{Evaluation}

We conducted a user study with two expert users to evaluate the design of EEGVis, where the first user is the one that we worked with 

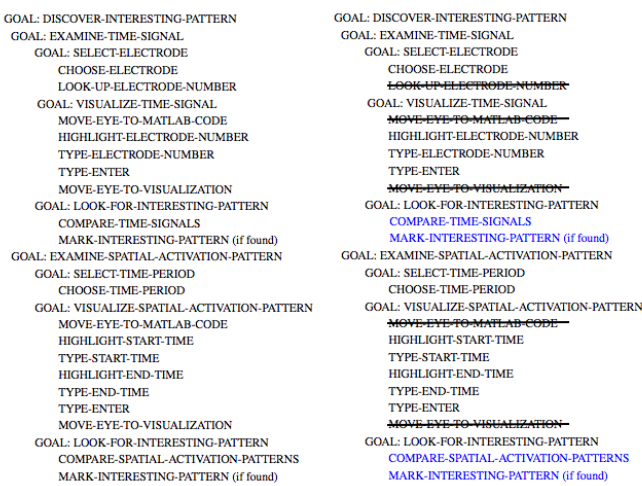

Figure 1: GOMS models for the user's standard workflow(left) and EEGVis workflow(right). With EEGVis, some wasted user actions from the standard workflow are significantly reduced (crossed out) and some tasks are less mentally demanding to perform (highlighted in blue).

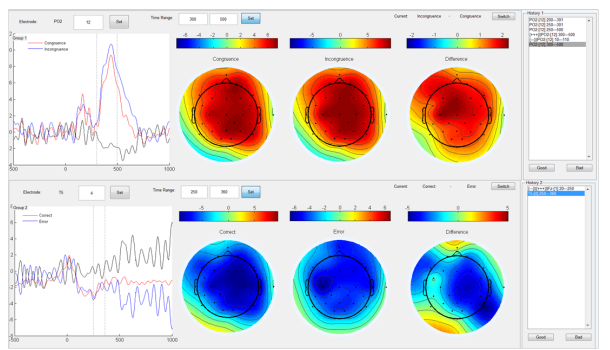

Figure 2: User Interface of EEGVis

to build the GOMS task model. To approximate real-world scenarios, each user was asked to choose a dataset to analyze and define his own analysis objectives. Each user analyzed his data through four separate sessions, using either the standard approach, i.e., a combination of Matlab and EEGLab, or EEGVis in each session. The four sessions are ordered as: Standard - EEGVis - EEGVis Standard. There was no time limitation: each user worked with the data until he felt there was no need to visually analyze the dataset further.

After each session, we asked the user to fill out a NASA Task Load Index [7] questionnaire to indicate his subjective cognitive load. After the user had finished all four sessions, we interviewed the user for his subjective feedback.

\section{Results}

The questionnaire scores are summarized in figure 3, where the scores for each workflow are averaged across four sessions. The chart shows that the users' subjective cognitive loads are much lower using EEGVis than using the standard approach.

Both users provided positive feedback about EEGVis in the interviews. The first user said that EEGVis allowed him to "build a whole representation of the dataset and learn about the dynamics of the brain from it." and that "by using the tool to view the dataset, I will eventually be able to derive novel hypotheses and study them". The second user stated that with EEGVis "I don't have to constantly switch from the script to the visualizations or from one visualization to another." It also appears that the first user is more productive using EEGVis. While he spent around $8 \mathrm{~min}$ utes for each of the Standard session, he spent only about 4 minutes for the first EEGVis session. And while he spent 8 minutes in the second EEGVis session, he reported that he examined all the electrodes in that session, while usually he would only go through half of them using EEGLab, since "it is too painful to go through all the electrodes using EEGLab".

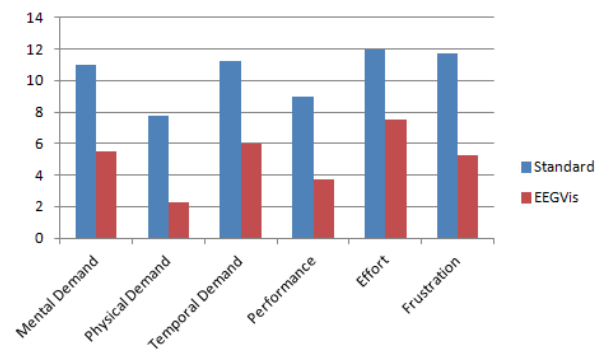

Figure 3: Questionnaire Results. A higher score indicates higher subjective cognitive effort.

\section{Discussion}

In addition to incorporating GOMS analysis into the design process, we also observe that each sub-goal in the task model suggests a benchmark task for evaluation. In this case, EXAMINE-TIME-SIGNAL and EXAMINE-SPATIAL-ACTIVATION-PATTERN could be used as benchmark tasks as these are essential components of the user's high-level goal and need to be supported by various EEG visual analysis tools.

One limitation of GOMS analysis for visual analysis tool design revealed in this study is that the model provides no mechanisms to represent the dependencies among sub-goals. For example, the output from EXAMINE-TIME-SIGNAL is not only interesting by itself but also sometimes serves as input to EXAMINE-SPATIAL-ACTIVATIONPATTERN as the user relies on the temporal signal to determine which time periods are interesting. It would be interesting to be able to capture the dependencies between subgoals in a task model as they might have implications on the importance of each task.

\section{CONCLUSION}

We have designed EEGVis, a visual analysis tool for EEG data based on GOMS analysis of the user's current workflow. Through the GOMS analysis, we were able to identify important user tasks as well as wasted user actions in the user's current workflow and use this information to make important design decisions. Evaluation results show that the new tool largely increases user performance and reduces users' subjective cognitive load compared to the original approach. In addition to building a tool that could potentially help researchers perform more comprehensive EEG data analysis with less effort, our case study also demonstrates an effective integration of GOMS analysis into the design process of a visual analysis tool.

\section{REFERENCES}

[1] R. Amar, J. Eagan, and J. Stasko. Low-level components of analytic activity in information visualization. In Information Visualization, 2005. INFOVIS 2005. IEEE Symposium on, pages 111 - 117, oct. 2005.

[2] S. K. Card, A. Newell, and T. P. Moran. The Psyc. of Human-Computer Interaction. L. Erlbaum Associates Inc., Hillsdale, NJ, USA, 1983.

[3] B. Lee, C. Plaisant, C. S. Parr, J.-D. Fekete, and N. Henry. Task taxonomy for graph visualization. In Proceedings of the 2006 AVI workshop on BEyond time and errors: novel evaluation methods for information visualization, BELIV '06, pages 1-5, New York, NY, USA, 2006.

[4] B. Shneiderman. The eyes have it: a task by data type taxonomy for information visualizations. In Visual Languages, 1996. Proceedings., IEEE Symposium on, pages 336 -343, sep 1996.

[5] S. Wehrend and C. Lewis. A problem-oriented classification of visualization techniques. In Proceedings of the 1st conference on Visualization '90, VIS '90, pages 139-143, Los Alamitos, CA, USA, 1990. IEEE Computer Society Press.

[6] Delorme, A. and Makeig, S. EEGLAB: an open source toolbox for analysis of single-trial EEG dynamics including independent component analysis. Journal of Neuroscience Methods 134, pages 9-21, 2004.

[7] Hart, S. G. and Staveland, L. E. Development of NASA-TLX (Task Load Index): Results of empirical and theoretical research. In P. A. Hancock and N. Meshkati (Eds.) Human Mental Workload. Amsterdam: North Holland Press, 1998 